\title{
Können Öko-Siegel einen neuen Handlungsspielraum für kleinbäuerliche Kaffeeproduzenten schaffen? - Das Beispiel Costa Rica
}

\section{Claudia Mayer, Eschborn}

\section{Einleitung}

Kaffee ist seit 1977 das zweitwichtigste Welthandelsgut nach Erdöl. Kaffeeanbau stellt die Lebensgrundlage für ca. 100 Millionen Menschen dar, von denen der weit überwiegende Teil in Ländern des «Südens» lebt (Sтамм 1999: 139). Bis in die 1960er-Jahre hinein wurde Kaffee noch im traditionellen Anbausystem erzeugt, bei dem der Kaffeestrauch unter einem mehr oder minder dichten Stockwerk aus Bäumen wächst. Diese Anbauweise wurde in vielen Gebieten durch die unbeschattete Monokultur von Hochleistungssorten abgelöst. Die Technisierung des Anbaus und die Ausbreitung von Monokulturen haben Auswirkungen auf den Rückgang der globalen Artenvielfalt und damit letztendlich auch auf die Regionen der Absatzländer. Gleichzeitig führte diese Entwicklung zu einer strukturellen Überproduktion, die zu stetig sinkenden Preisen der Kaffeebohnen und einer Verarmung der kleinbäuerlichen Kaffeeproduzenten führt (RICE \& WARD 1997; Commission for Environmental Cooperation (CEC) 1999; United Nations Conference on Trade and Development (UNCTAD) 1993; Boyce et al. 1994; Gomez-Pomba 1997; Gommans 1996; Vásouez 1999).

Kaffee ist zudem ein Produkt, das aufgrund seiner klimatischen Anforderungen nicht in den Konsumregionen angebaut werden kann. Die Verbraucher in den Konsumländern wollen auf die schwarzen Bohnen, die Bestandteil des alltäglichen Lebens geworden sind, nicht mehr verzichten. Gleichzeitig ist ihre Sensibilität für umwelt- und entwicklungspolitische Belange gestiegen und damit die Nachfrage nach ökologisch produziertem Kaffee geschaffen worden. Der Handel greift entsprechend nicht auf Substitute zurück, sondern sucht nach umweltverträglich angebautem Kaffee. Umweltsiegel auf der Produktpackung sollen dem Konsumenten die umweltverträgliche Produktion des Kaffees und dem Produzenten eine Steigerung der Wettbewerbsfähigkeit durch den Eintritt in eine wachsende Marktnische gewährleisten. $\mathrm{Ob}$ die veränderten Nachfragepräferenzen und die dadurch kreierte neue Marktnische in einem übersättigten Kaffeeweltmarkt einen neuen Handlungsspielraum für die Kaffeebauern darstellen, soll am Beispiel Costa Rica dargestellt werden.

\section{Zielsetzung und Methode}

Der vorliegende Artikel basiert auf Auswertungen von Leitfrageninterviews, die mit Akteuren entlang der Warenkette von Kaffee sowie involvierten Organisationen innerhalb des Forschungsprojekts «Umweltbezogene Produktstandards und ihre Umsetzung in der Kaffee- und Bananenproduktion» geführt wurden, das Teil des Schwerpunktprogramms der Deutschen Forschungsgemeinschaft (DFG) «Mensch und globale Umweltveränderungen» am Fachbereich Geographie der Philipps-Universität, Marburg war. Diese Untersuchung basiert auf der Hypothese, dass die Signale, die von den großen Märkten des Nordens ausgehen, nur dann umweltrelevante Innovationen auslösen, wenn sie die Anpassungsfähigkeit der Kaffeeproduzenten nicht überfordern, d.h. die Kaffeebauern den durch die Umweltsiegel neu geschaffenen Handlungsspielraum auch nutzen können.

Den Grundgedanken, dass eine Änderung der Produktionsweise - in diesem Fall ein Wandel zu einer ökologischen Produktionsweise - durch die Nachfrage angeregt werden könnte, greifen GerefFI, KORZENIEWICZ \& KoRZENIEWICZ (1994) in ihrem Ansatz der Global Commodity Chain (GCC) auf. In diesem Ansatz wird von strategischen Schlüsselstellen ausgegangen, die es erlauben, eine Warenkette zu dominieren, d.h. die Regeln für alle anderen Akteure vorzugeben. Diese Vorgaben bestimmen nicht nur, was produziert wird, sondern auch über die Art und Weise der Produktion, also auch die Umweltverträglichkeit der Produktionsweise sowie über die Verteilung des Mehrwerts entlang der Kette. Der jeweilige Zugang zu Ressourcen, die Organisationsform und die Rahmenbedingungen bestimmen die Handlungsbedingungen der jeweiligen Akteure, d.h. die Handlungs- und Entscheidungsspielräume der Akteure (GEREFFI, KoRZEWIENICZ \& KORZEWIENICZ 1994; HopKINS \& WALLERSTEIN 1994; TALBot 1997a und 1997b; vgl. RaIKeS et al. 2000). Die Rahmenbedingungen stecken dabei die Grenzen der Handlungsspielräume für Individuen $\mathrm{ab}$, die individuelle Gestaltung des Spielraumes aber bleibt offen. Ob dieser Handlungsspielraum durch entsprechende Handlungsstrategien zum Handlungsraum wird, hängt von bestehenden Anreizen und Hindernissen sowie der Befähigung der Individuen ab, ihre Handlungsstrategien an gegebene Rahmenbedingungen anzupassen.

Im Kaffeesektor bedeutet dies, dass veränderte Rah- 


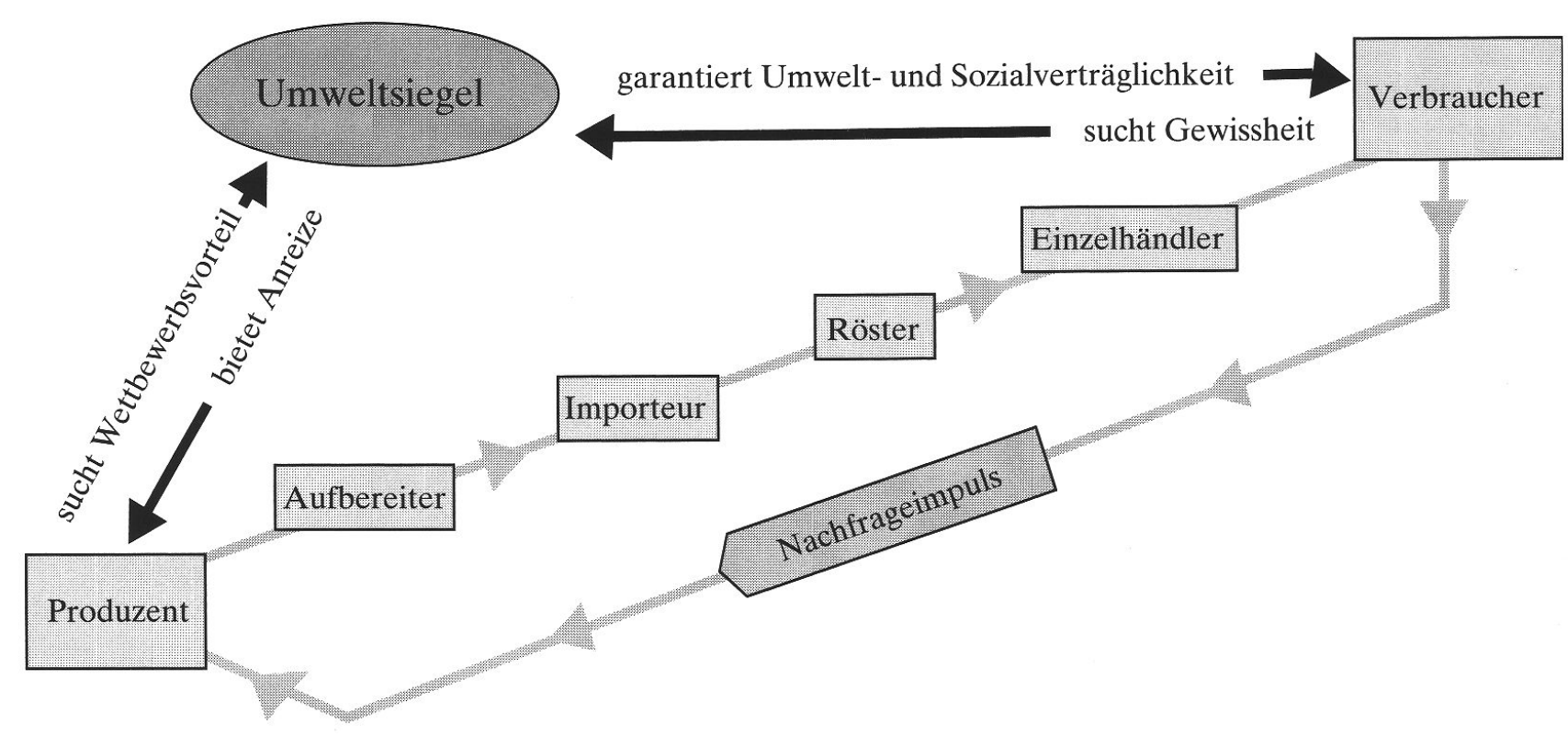

Abb. 1: Brückenfunktion des Umweltsiegels zwischen Verbraucher und Produzent Environmental lables as a link between consumer and producer Fonction de pont du label environnemental entre consommateur et producteur Entwurf: C. MAYER

menbedingungen, wie sie durch die Existenz von ÖkoSiegeln geschaffen wurden, den Produzenten eine Steigerung ihrer Wettbewerbsfähigkeit bei gleichzeitigem Erhalt ihrer natürlichen Produktionsgrundlagen ermöglichen sollen. Erforderlich hierfür ist eine entsprechende Befähigung der Produzenten. Ob das ÖkoSiegel als veränderte Rahmenbedingung einen ausreichenden Handlungsspielraum schaffen kann und ob die Produzenten diesen nutzen können, soll im Folgenden am Beispiel der Kaffeebauern Costa Ricas dargestellt werden.

\section{Handlungsspielräume durch veränderte Nach- fragepräferenzen auf dem Weltkaffeemarkt}

Das weltweite Handelsvolumen von ökologisch angebautem Kaffee weist hohe Wachstumsraten von jährlich $15-18 \%$ auf. Die USA mit $44,8 \%$ und Deutschland mit 35,8\% stellen weltweit die Hauptimporteure von ökologisch angebautem Kaffee dar (GiovanNUCCI 2001: 23ff.; Rosen et al. 2000; Rice \& McLean 1999: 72). Am US-amerikanischen Kaffeemarkt hatte der ökologische Kaffee 1996 mit einem Umsatz von 50 Millionen US\$ einen Marktanteil von 2\%. Dieser bescheidene Anteil verzeichnete jedoch ein rasantes Wachstum: 1999 konnte bereits ein Handelsvolumen von 100-150 Millionen US\$ und damit 4\% am US-amerikanischen Kaffeemarkt erzielt werden (FISCHERSWORRING 2001: 145; Commission fOR Environmental CoOperation
(CEC) 2000:12; Rodriguez 2001:16f.; Rosen et al. 2000: 4). Hohe Wachstumsraten der Nachfrage nach ÖkoKaffee zeigen sich auch in Deutschland, wo der Marktanteil von knapp einer Million pound $(=0,454 \mathrm{~kg})$ importiertem ökologischem Kaffee 1997 mit 74 Mio. DM 2\% des Gesamthandelswertes von ökologischen Produkten betrug (International Trade OrganizaTION (ITC) 1999: 55f., 179; BUNDESVERBÄNDE NATURKost Naturwaren (BNN) 2001:3f.).

Die steigende Nachfrage nach ökologischen Produkten im Allgemeinen und nach ökologischem Kaffee im Besonderen verstärkte in den Konsumländern die Forderung nach gesetzlich festgelegten Standards und Kontrollverfahren, um den Missbrauch mit Umweltsiegeln zu reinen Marketingzwecken zu reduzieren. Die gesetzlich niedergeschriebenen Umweltstandards für kontrolliert biologischen Anbau (kbA) sollen mittels einer Kennzeichnung von Kaffee den Konsumenten die ökologische Produktion garantieren. Den Produzenten soll der Zugang zu Marktnischen ermöglicht und die Anstrengungen für eine umweltverträgliche Produktionsweise durch einen Mehrpreis entlohnt werden. Ein Umweltsiegel soll die Kommunikation über die Produktionsweise zwischen dem Produzenten und dem Konsumenten gewährleisten (siehe Abb.1).

In der Europäischen Union (EU) ist seit 1993 die «Verordnung (EWG) Nr. 2092/91 über den ökologischen Anbau und die entsprechende Kennzeichnung der 


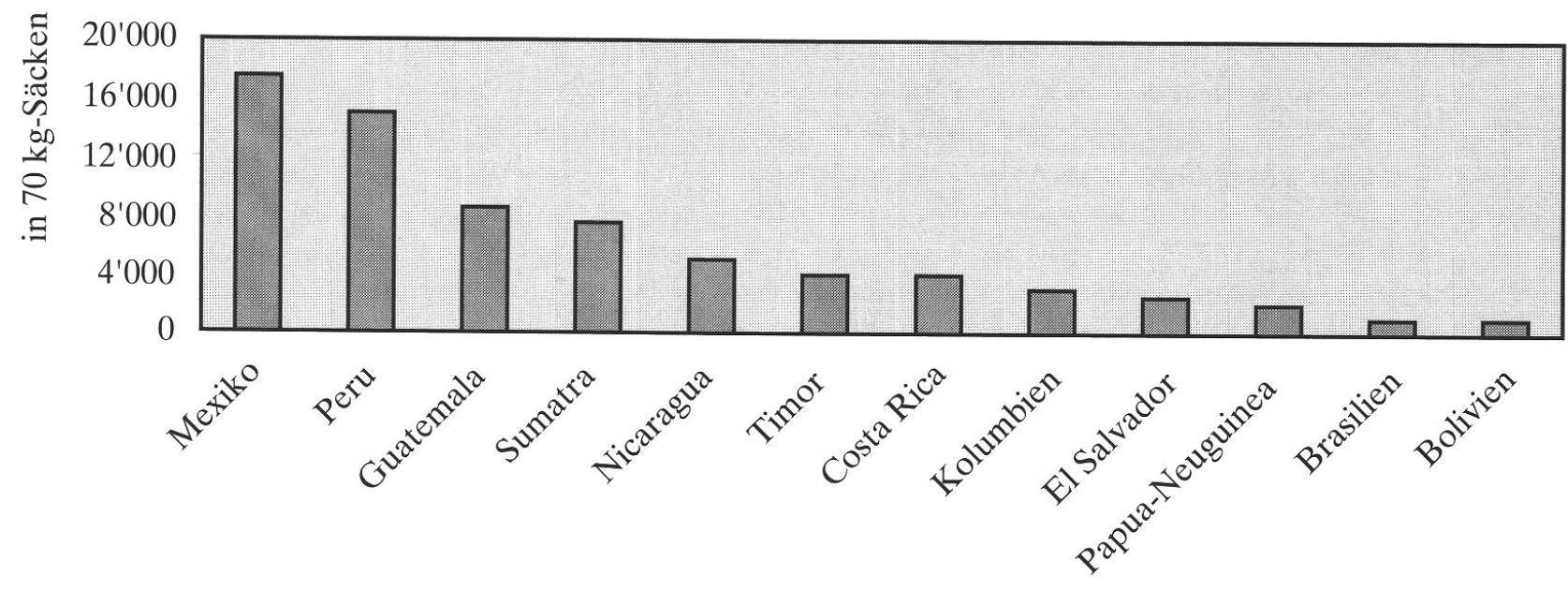

\begin{abstract}
Abb. 2: Produktionsregionen von ökologisch produziertem Kaffee für den Export nach den USA Areas producing organic coffee for the export market to the USA

Régions productrices de café produit écologiquement, en vue de l'exportation vers les USA

Quelle: Organic Coffee Association (ORCA, 2000) (veränd.)
\end{abstract}

landwirtschaftlichen Erzeugnisse und Lebensmittel» (EG-Öko-VO) rechtswirksam. Diese Verordnung definiert für alle Mitgliedsstaaten der Europäischen Union die Anforderungen, die eingehalten werden müssen, wenn ein Produkt mit Hinweisen auf den ökologischen Anbau vermarktet werden soll. In den USA trat durch das United States Department of Agriculture (USDA) im Frühjahr 2001 eine gesetzliche Regelung in Kraft.

Voraussetzung für die Ausstellung des Prüfsiegels, die Zertifizierung, ist eine Kontrolle, ob die Standards eingehalten wurden, die sogenannte Konformitätsprüfung. Kontrolliert wird die Einhaltung der Anforderungen durch unabhängige Dritte, die ihrerseits auf ihre Qualifikation geprüft und für dieses Verfahren akkreditiert werden. Hauptforderung der Standards für kontrolliert ökologischen Landbau ist eine dreijährige Umstellungszeit, in welcher keine synthetischen Düngeund Schädlingsbekämpfungsmittel eingesetzt werden dürfen. Nach den drei Jahren bleibt der Einsatz von synthetischen Dünge- oder Spritzmitteln verboten. Die gesamte Produktionskette wird einer Kontrolle unterzogen, um eine etwaige Kontaminierung des Produktes zu verhindern.

Die Produktion von ökologisch angebautem Kaffee ist international sehr ungleich verteilt (vgl. Abb. 2). Bei einigen Provenienzen und Qualitäten herrscht bereits ein Angebotsüberhang auf dem Weltmarkt vor, in Ursprungsgebieten von qualitativ sehr hochwertigem Kaffee wie in Costa Rica liegt hingegen ein Nachfrageüberhang vor. Kaffeeimporteure bezogen, insbesondere zur Qualitätssicherung ihres ökologischen Kaffees, Bohnen aus Kolumbien, Guatemala,
Mexiko und Costa Rica. Dabei betonten sie jedoch den vorherrschenden Angebotsengpass von ökologisch zertifiziertem Kaffee aus Costa Rica (vgl. RICE \& McLean 1999: 47; Rodriguez 2001: 15).

In Costa Rica waren 1999/2000 614 ha Kaffeefelder ökologisch zertifiziert. Dies entspricht einem Anteil von $0,6 \%$ der insgesamt 107.000 ha mit Kaffee bewirtschafteten Flächen (United States Department of Agriculture (USDA) - Global Agriculture InforMATION NeTWORK 2000). Die ökologisch zertifizierten Kaffeefelder befinden sich vorwiegend in Regionen mit suboptimalen Kaffeeanbaubedingungen, in denen relativ niedrigere Preise für ihre Kaffeebohnen erzielt werden. Insgesamt handelt es sich bei allen Innovatoren um Kleinproduzenten mit durchschnittlich 5 ha Anbaufläche. Lediglich zwei Großbetriebe mit 35 ha und einer mit 130 ha Anbaufläche bilden dabei eine Ausnahme. Gründe, warum bislang nur wenige und vorwiegend kleine Produzenten umgestellt haben, lassen sich sowohl in den vorherrschenden Rahmenbedingungen wie auch in einer unzureichenden Befähigung der Akteure finden (vgl. Abb. 3 und 4). Einige ausgewählte Aspekte sollen im Folgenden skizziert werden

\section{Begrenzungen des Handlungsspielraums durch existierende Rahmenbedingungen}

Eine Begrenzung des Handlungsspielraums ergibt sich zunächst aus dem Zertifizierungssystem selbst sowie aus den Anforderungen der Standards, die die Anpassungsfähigkeit der Produzenten in Teilbereichen überfordern. 

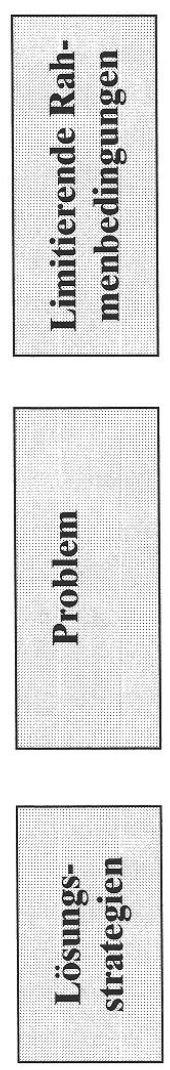

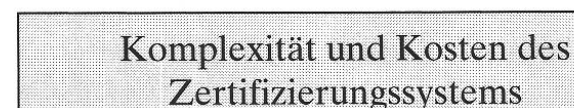

Zertifizierungssystems

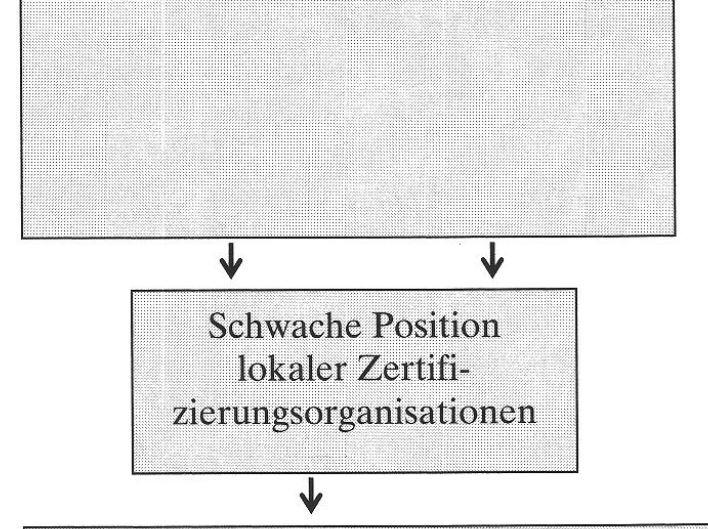

Begrenzung des Handlungsspielraumes

$\Downarrow$

$\Downarrow$

Stärkung der lokalen Kontrollstellen
Unzureichende

Berücksichti-

gung ökonomi-

scher Rahmen-

bedingungen
Unzureichende

Berücksichtigung regionalspezifischer ökologischer

Rahmenbedingungen

\begin{tabular}{|c|c|}
\hline Begrenzung des Handlungsspielraumes \\
$\qquad \begin{array}{c}\Downarrow \\
\text { Stärkung der lokalen Kontrollstellen }\end{array}$ \\
$\begin{array}{c}\text { Aufbau von Indikatoren für regionale } \\
\text { Gegebenheiten sowie für gerechte } \\
\text { Handelsbeziehungen }\end{array}$ \\
\hline
\end{tabular}

Abb. 3: Begrenzungen des Handlungsspielraums durch Öko-Siegel

Limitations on field of action by environmental label

Limitations de la liberté d'action par les labels écologiques

Entwurf: C. MAYER; Bearbeitung: N. AMMANN

Die Schwierigkeiten in der Anwendung der kbAStandards liegen nach Aussagen der Befragten nicht nur in den Anforderungen der Standards selbst, sondern ebenfalls im Kontroll- und Zertifizierungsprozess. Trotz der EU-Verordnung haben einzelne europäische Länder unterschiedliche Verfahren der Anerkennung von Zertifizierungsorganisationen etabliert und die gegenseitige Anerkennung der jeweils akkreditierten Organisationen befindet sich noch im Prozess. Diese unterschiedlichen Verfahren in einzelnen europäischen Ländern und den USA erfordern eine hohe Kenntnis der jeweiligen Anforderungen und Verfahren. Möchte ein Produzent seinen Öko-Kaffee auf mehreren Absatzmärkten verkaufen, entstehen ihm zusätzliche Kosten durch eine Doppel- bis Dreifachzertifizierung, wenn unterschiedliche Zertifizierungsorganisationen für die jeweiligen Länder akkreditiert sind. Ein kostenerhöhender Faktor ist auch, dass nach wie vor aus den Konsumländern stammende international tätige Organisationen die Kontrolle ausführen, und damit höhere Reisekosten anfallen. Nationale Kontrollorganisationen haben bislang einen komparativen Wettbewerbsnachteil, da sie oftmals die teuren Akkre- ditierungskosten nicht finanzieren können und zudem über weniger Erfahrung und Kontakte in diesem Markt verfügen. Die Zertifizierungskosten stellen für die Produzenten, insbesondere in der Umstellungszeit, eine Hürde dar, weil sie vor allem in dieser Anfangszeit mit Mindererträgen von bis zu 50\% rechnen müssen, ohne einen Mehrpreis für ihr Produkt zu erhalten. Erst einige Jahre nach der Umstellung, so die Aussagen der Produzenten, gehen mit der Regenerierung des Ökosystems auch Ertragssteigerungen einher (Luibrand 1997; CoRPoración EduCativo PaRa el Desarollo Costarricense (CEDECO) 1999; LyNBAEK 2000).

Nach der Umstellungszeit erhielten die befragten Produzenten in Costa Rica einen Mehrpreis zwischen 60 und 160 US-Cents/kg. Ob damit ein Anreiz zur Produktion von ökologisch zertifiziertem Kaffee besteht, hängt in hohem Maße von der Rentabilität der Anbauweise ab. Vergleichsstudien zur ökonomischen Rentabilität von konventionellem und ökologischem Anbau in Costa Rica (AKKerman \& VAN BAAR 1992; Boyce et al. 1994; Alvarado Soto 1996; Luibrand 


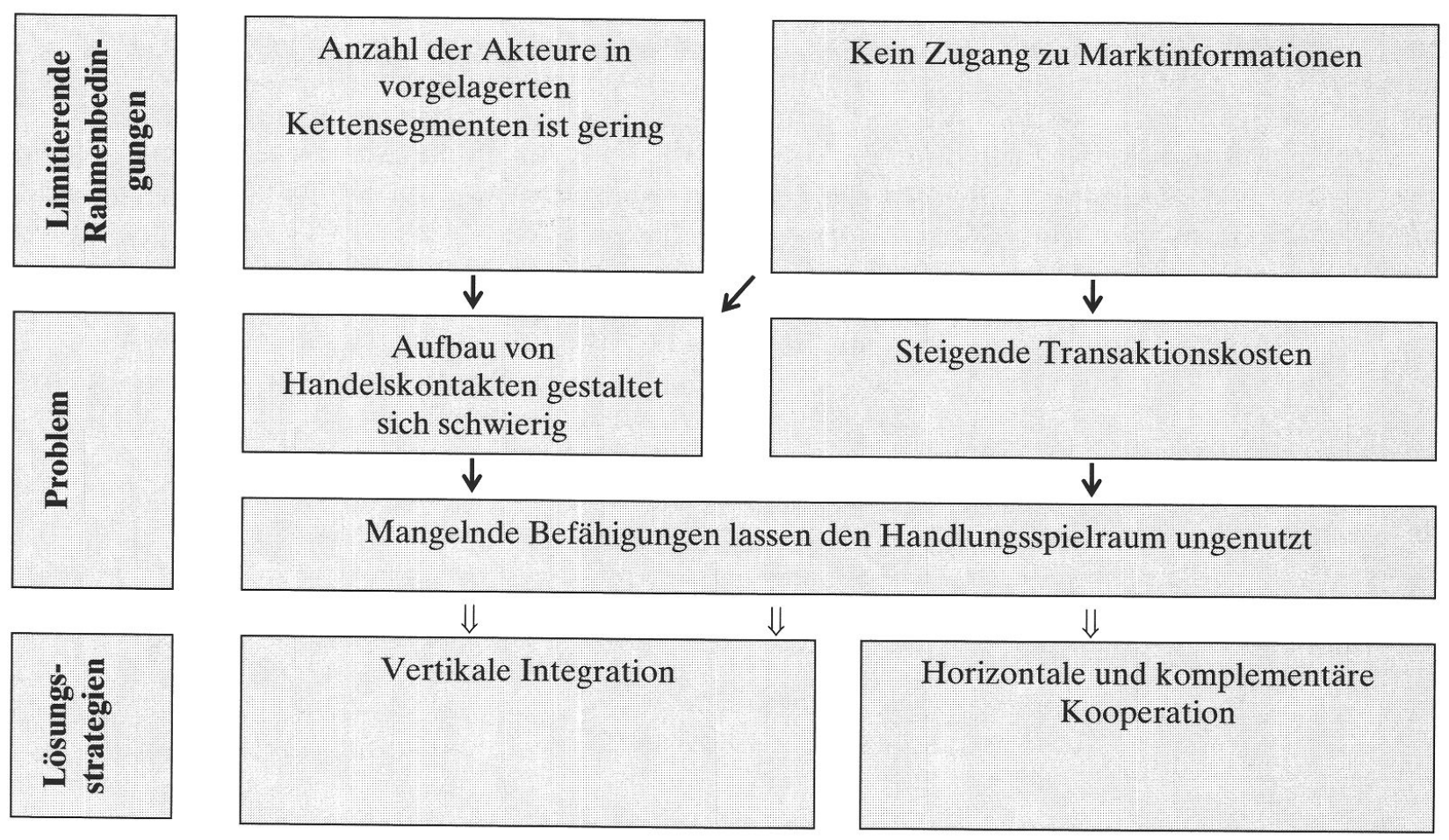

Abb. 4: Unzureichende Befähigung der Akteure und Lösungsstrategien Inadequate qualifications of the actors and strategies to solve the problem Insuffisante qualification des acteurs et solutions stratégiques Entwurf: C. MAYER; Bearbeitung: N. AMMANN

1997; Corporación Educativo para el Desarollo Costarricense (CEDECO) 1999; Lynbaek 2000) ergaben für die Produzenten im ökologischen Anbau ein um 13 bis $45 \%$ geringeres Nettoeinkommen. In Abhängigkeit der Faktoren Bodenqualität und Höhenlage variieren die Durchschnittserträge von ökologisch angebautem Kaffee zwischen 10 und 30 Quintal/ha (1 Quintal = 46,6 kg). Beim konventionellen Anbau liegt der durchschnittliche Ertrag zwischen 50 und 60 Quintal/ha, allerdings mit einer Spanne zwischen 15 und 100 Quintal/ha je nach Region. Insgesamt kommen die Studien zu dem Ergebnis, dass trotz höherer variabler Kosten, insbesondere der Arbeitskosten, die Gesamtkosten im ökologischen Anbau bis zu 20\% niedriger ausfallen. Die geringeren Erträge und damit schwächeren Umsätze in US\$/ha werden durch die erzielten Mehrpreise nicht ausgeglichen. Aus diesem Grund sind es vorwiegend kleine Betriebe, die umstellen. Diese können von den sinkenden Kapitalkosten durch den Verzicht auf synthetischen Dünger und Pestizide profitieren, der Mehraufwand an Arbeitskraft wird nicht kalkuliert, weil sie fast ausschließlich mit Familienarbeitskräften operieren. Den höchsten Wertschöpfungsanteil erzielten auch im ökologischen Anbau die Kaffeeröster (MAYER 2002; vgl. TAlbot 1997a).
Neben den ökonomischen Risiken sind die Kaffeebauern auch mit den Anforderungen der Standards selbst konfrontiert (vgl. Abb. 3). Das Siegel des ökologischen Anbaus verbietet jeglichen Einsatz von synthetischen Dünge- und Spritzmitteln und fordert damit implizit die Wiedereinführung von Schattenbäumen als Nährstoffeinträger. Ein an die naturräumlichen Bedingungen nicht angepasster Schatten, d.h. ein Schatten, der durch zu starke oder zu geringe Beschattung und/oder zu geringen Abstand der Baumkrone zur Kaffeepflanze nicht entsprechend als Temperatur- und Feuchtigkeitsregler funktionieren kann, schafft suboptimale bis mangelhafte Bedingungen für die Kaffeepflanzen (GALLOWAY \& BeER 1997; Beer et al. 1998; Muschler 1999, 1997; Espinoza 1985: 145). Das unzureichende Wissen über standortgerechten Schatten wurde aber von $30 \%$ der Produzenten in Costa Rica als Haupthemmnis für eine Umstellung angegeben (CORPORACIÓN EDUCAtivo para el Desarollo Costarricense (CEDECO) 1999). Insbesondere in den über $1.200 \mathrm{~m}$ über NN gelegenen Kaffeeanbauregionen sowie jenen mit suboptimalen Bedingungen, die erst im Rahmen der Flächenexpansion und von Beginn an intensiv bewirtschaftet wurden, liegt in Costa Rica kein traditionelles Erfahrungswissen vor, und Forschungen hierzu laufen erst in jüngster Zeit an. 
Um diese den neuen Handlungsspielraum begrenzenden Rahmenbedingungen zu reduzieren, sind Bemühungen zu intensivieren, um nationale Kontrollstellen aufzubauen und einheimische Fachkräfte für diese Tätigkeit auszubilden. Um das ökonomische Risiko zu reduzieren und einen Anreiz für die Produzenten zu schaffen, sollten in die Standards für kontrolliert biologischen Anbau auch Standards für gerechte Handelsstrukturen einfließen, die von externen und neutralen Kontrollorganisationen zu prüfen sind. Eine gerechtere Verteilung der Wertschöpfung und damit ein ökonomischer Anreiz muss gewährleistet werden, damit mittels des Öko-Siegels eine Verbesserung der Lebenssituation von kleinbäuerlichen Kaffeeproduzenten erreicht werden kann. Den Herausforderungen hinsichtlich der Anbautechniken muss wegen der Schwierigkeit, ein vielfältiges Ökosystem kleinräumig zu systematisieren, mit verstärkten Forschungsanstrengungen begegnet werden, um regionalspezifische Indikatoren für standortgerechte Schattenbaumdichten und -arten festlegen zu können. Insbesondere dann, wenn die staatliche Politik Forschungen zu standortgerechtem Anbau vernachlässigt und nach wie vor an der Strategie der Produktivitätssteigerung festhält, mangelt es den Produzenten an Wissen über standortgerechten Schatten. In partizipativen Forschungsworkshops, in denen die Erfahrungen der Produzenten in die Erarbeitung von regionalspezifischen Indikatoren integriert werden, liegt ein bislang vernachlässigtes Potenzial. Erste Ansätze - induziert von Nichtregierungsorganisationen (NGOs) -, ein Netzwerk zwischen Produzenten zum Erfahrungs- und Wissensaustausch zu organisieren, sind darauf orientiert, das Expertenwissen der Produzenten sinnvoll zu nutzen.

\section{Begrenzung des Handlungsspielraums durch unzureichende Befähigung der Akteure}

Aufgrund der geringen Anzahl von Akteuren im Segment von öko-gesiegeltem Kaffee und des allgemein schwierigen Informationszugangs über veränderte Marktstrukturen in ländlichen Gebieten gestalten sich die Kosten für die Suche nach neuen Handelsbeziehungen hoch (vgl. Abb. 4.). Diese sind jedoch notwendig, da sich alle Akteure der Warenkette einer Kontrolle unterziehen müssen, soll der Kaffee das kbA-Siegel erhalten.

Das Risiko neuer Handelsbeziehungen ist groß, wenn zwei Partner in der Kette eine ökologische Optimierung ihrer Produkte anstreben. Riskant ist, wenn sich beispielsweise der Produzent auf die spezifischen Interessen des Verarbeiters einlässt und seine gesamte Produktion in der Erwartung umstellt, dass der Verarbeiter seine Absprachen einhält und einen entsprechenden Mehrpreis zahlt. Verstärkt wird das Risiko, wenn keine alternativen Abnehmer existieren, um die ökologischen Kaffeebohnen als solche und $\mathrm{zu}$ äquivalenten Preisen aufzukaufen. Hält sich der Verarbeiter nicht an die Absprachen, ist der Produzent gezwungen, seine Ernte als konventionelle zu verkaufen und Verluste hinzunehmen. Aus diesem Grund erfährt die Art und Weise der Kooperationsform einen Bedeutungszuwachs. Entweder werden langfristige Handelsverträge geschlossen oder eine vertikale Integration entlang der Produktionskette vorgenommen. Die Handlungsstrategie der vertikalen Integration der Produktionskette nutzen die Akteure insbesondere, um das Defizit an vor- und nachgelagerten Kettensegmenten auszugleichen und/oder das erhöhte Risiko der Zulieferer- und Abnehmerbeziehungen aufgrund der begrenzten Verfügbarkeit der gesiegelten Ware zu verkleinern (vgl. Abb. 5).

In den untersuchten Beispielen konnten die Produzenten durch den Prozess der vertikalen Integration der Abschöpfung durch vorgelagerte Segmente entgegenwirken und durch die Kontakte mit den Röstern einen direkten Kommunikationskanal für ein Qualitätsmanagement aufbauen. Die geringe Anzahl der Akteure birgt hierbei den Vorteil, dass sich die Mehrheit der Akteure durch Veranstaltungen, Messen und Konferenzen persönlich kennen. Die Risiken der Integration liegen in den hohen Organisationsund Lernkosten, da Erfahrungen, Handelskontakte und Zugang zu Marktinformation für die Verhandlungen nötig sind (vgl. Porter 2000: 123). Nicht zu vernachlässigen sind die Kosten zum Aufbau von Vertrauen. Übernimmt ein Produzent erstmals die Funktion eines Exporteurs, ist er zunächst eine unbekannte Größe und muss Reputation erst aufbauen. Um Schwierigkeiten begegnen zu können wie die, eine ausreichende Menge für den Direktexport zu produzieren und die Kosten für die Zertifizierung zu tragen, sind horizontale Kooperationen zwischen gleichen Kettensegmenten erforderlich.

Horizontale Kooperationen sind Netzwerke, auch sogenannte strategische Allianzen, von Unternehmen gleicher Segmente der Warenkette mit dem Ziel, die eigene Wettbewerbsfähigkeit bzw. Marktmacht zu stärken. Durch Zusammenschlüsse können Zertifizierungskosten minimiert werden, indem sie eine Gruppenzertifizierung beantragen, deren Kontrollaufwand im Verhältnis zur Einzelbetriebskontrolle wesentlich kleiner ist. Die meisten Kontrollorganisationen prüfen bei einer Gruppenzertifizierung 20-50\% der Mitglieder. Die Zertifizierungskosten, die bis zu $10 \%$ des erhaltenen Mehrpreises je pound $(=0,454 \mathrm{~kg})$ betragen, können dadurch erheblich reduziert werden. Als eine Art eines strukturell erzwungenen Zusammenschlusses beschreibt RodrigueZ diese Form des Organisierens, wenn er sagt: 


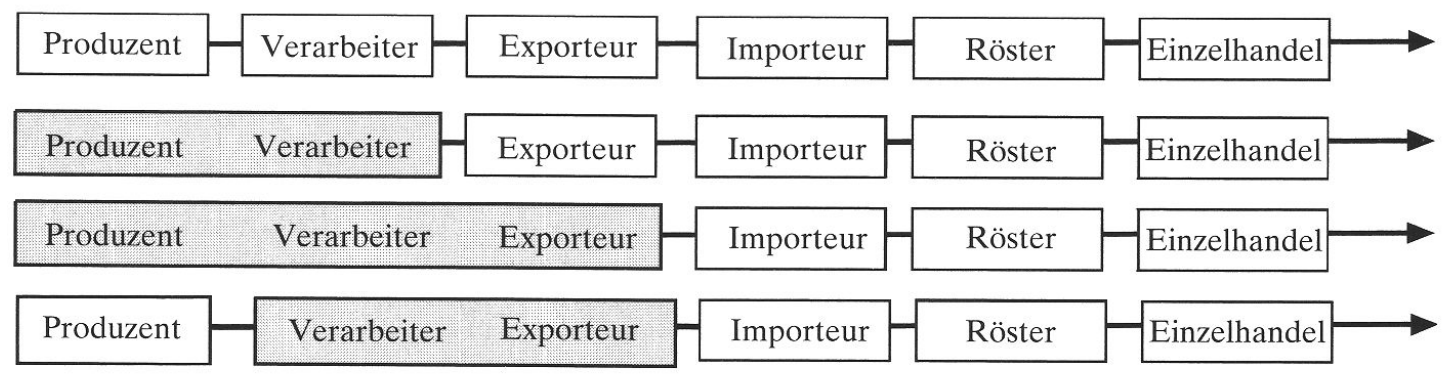

vertikal integrierte Warenkettensegmente

\begin{abstract}
Abb. 5: Grad der vertikalen Integration der Warenkette von Kaffee im Produktionsland Degree to which the product chain of coffee is vertically integrated in its country of production Degré d'intégration vertical de la chaîne de marchandises du café dans le pays producteur Entwurf: C. MAYER
\end{abstract}

«Because of the expense of certification, producers, largely small landholders, are driven to unite in a cooperative manner in order to spread the cost over several members» (2001: 17).

Forciert wurde der Organisationsprozess der Produzenten in starkem Maße allerdings auch von den Verarbeitern, die von niedrigen Zertifizierungskosten profitieren, da sie in der Regel die Kostennehmer sind und die Produzenten so zu einer Abliefergarantie verpflichten. Sind die Zertifizierungskosten aber gesenkt, können sie von Produzentengruppen selbst übernommen werden und damit können neue Abhängigkeiten durch das sogenannte Andienungsabkommen reduziert werden.

Ein weiterer Vorteil des Zusammenschlusses ist im Zusammenhang mit der vertikalen Integration der Warenkette zu sehen. Will ein Produzent auch die Funktion des Exporteurs übernehmen, benötigt er für den Direktexport eine Menge von 17.250 kg Kaffee, was der Füllmenge von einem Container und einer Fläche von ca. 10 ha entspricht. Da Kleinproduzenten in der Regel nicht über mehr als 3 ha verfügen, ist eine Kooperation mit anderen Anbietern notwendig, um die erforderliche Menge zu erreichen.

Um den Schwierigkeiten und Hürden dieser Lernprozesse, insbesondere in der Umstellungszeit, zu begegnen, zeigten sich komplementäre Kooperationsformen als unerlässlich. Dieser Form der Zusammenarbeit von Kaffeebauern mit überbetrieblichen Organisationen wie Verbänden, Forschungseinrichtungen, politischen Einrichtungen und Nichtregierungsorganisationen sowie der internationalen Zusammenarbeit wird eine große Bedeutung zugesprochen (CourviLle 2001; Ponte 2001; Giovanucci 2001; Rice \& McLean 1999). Dies gilt wiederum in besonderem Maße, wenn die staatliche Infrastruktur keine förderlichen Rahmenbedingungen für eine Umstellung auf eine ökologische
Produktionsweise geschaffen hat und die Banken ihre Kreditkonditionen nicht selten an den Einsatz des technologischen Paketes binden (RICE \& MCLEAN 1999: 24.). Bis heute haben nur wenige Kreditgeber die Chancen erkannt, die in der Marktnische des ökologischen Kaffeeanbaus verborgen sind. Ohne Kredite wird die Umstellung zu einer erfolgskritischen Phase und nicht wenige Produzenten mussten hochverschuldet wieder zum konventionellen Anbau zurückkehren.

Die Entwicklungszusammenarbeit stellt eine dieser komplementären Kooperationsformen dar, die auf institutionelle Rahmen- sowie direkte Handlungsbedingungen der Akteure der Warenkette Einfluss nimmt. Zahlreiche Aktivitäten zielen auf die Stärkung der jeweiligen nationalen Rahmenbedingungen ab. Dazu gehören beispielsweise:

- die Beratung bei der Erarbeitung von neuen Gesetzen,

- die personelle Unterstützung der (inter-)nationalen auf ökologischen Kaffeeanbau spezialisierten Forschungsinstitute, oder

- die Förderung nationaler Zertifizierungsorganisationen.

Direkte Handlungsbedingungen werden in Form von Veranstaltungen, Seminaren und Workshops über ökologische Anbaumethoden in der Landwirtschaft und deren Vermarktung angeboten. Bis 1998 förderte die Deutsche Gesellschaft für Technische Zusammenarbeit (GTZ) eine Verkaufsmesse für ökologische Produkte in der Hauptstadt Costa Ricas und lädt nach wie vor auch Produzenten zur weltweit bekannten Fachmesse «Biofach» in Deutschland ein. Durch den Aufbau von Informationsnetzwerken wie das «Green-Trade-Net» (www.green-tradenet.de ) soll insbesondere den Kleinproduzenten der gebührenfreie Zugang zu Auskünften über sektor- und produktspezifische Handelskontakte und das Marktgeschehen ermöglicht werden. 


\section{Fazit}

Der durch das kbA-Siegel neu geschaffene Handlungsspielraum ist durch Rahmenbedingungen und unzureichende Befähigung der Akteure noch nicht genügend genutzt. Es zeigt sich, dass sich der Markt nicht selbst reguliert, um eine verbesserte Lebenssituation der Kaffeeproduzenten zu erreichen. Nur wenn das Siegel eine Optimierung in dem Sinne erreicht, dass die Produzenten nicht nur zum Eintritt in die Marktnische, sondern auch zur eigenständigen Teilhabe am Weltmarkt befähigt werden, ihnen eine Finanzierung ermöglicht und Beratung bereitgestellt wird, kann Kaffeeanbau mit Öko-Siegel nachhaltig sein. Funktionierende Kooperationen zwischen den Akteuren entlang der Warenkette stellen dabei eine sinnvolle und teilweise notwendige Ergänzung des Öko-Siegels dar. Es zeigte sich, dass diejenigen kleinbäuerlichen Kaffeeproduzenten, die ihre Handlungsstrategien unter den gegebenen Handlungsbedingungen verändert haben, von den Öko-Siegeln profitieren konnten. Trotzdem sollte die Forderung, an die ökologischen Standards auch soziale Standards zu koppeln, bestehen bleiben. Ohne derartige institutionelle Absicherungen und entsprechende Kontrollmechanismen durch neutrale «Dritte» oder «Vierte» sind Kollektiv-Vereinbarungen zwischen Akteuren durch unvollständige und asymmetrisch verteilte Kenntnisse sowie unfaire Spielregeln des Marktwettbewerbs der Gefahr ausgesetzt, von Einzelinteressen unterwandert zu werden. Werden den Machtungleichgewichten keine institutionellen Grenzen gesetzt, kann, wie BARTMANN es provokativ formuliert, von «Verhandlungslösungen als Schönwetterveranstaltung» gesprochen werden (BARTMANN 1999: 6).

Damit das Öko-Siegel einen Handlungsspielraum für kleinbäuerliche Kaffeeproduzenten schaffen kann, bedarf es Entwicklungsstrategien, die an den Rahmen- bzw. Handlungsbedingungen wie auch an der Befähigung der Akteure anknüpfen. Der isolierte Versuch, die Bedingungen menschlichen Handelns durch eine Erweiterung der Handlungsspielräume zu beeinflussen und die Zertifizierung mit dem Öko-Siegel vorzunehmen, führen nur dann zum Erfolg, wenn die Betroffenen finanziell wie informationell befähigt sind, die neuen Spielräume auch zu nutzen.

\section{Literatur}

AkKerman, A. \& P. van BaAR (1992): El café orgánico: la sostenibilidad de un grano de oro. - San José, Amsterdam.

Alvarado Soto, M. (1996): Estudio comparativo de dos sistemas de producción de café: convencional y orgánico. - San José: Universidad Nacional.

Bartmann, H. (1999): Kooperationslösungen aus umweltökonomischer Sicht. $-=$ Beiträge zur Wirt- schaftsforschung 61, Johannes Gutenberg-Universität Mainz; http://wiwi.uni-mainz.de/vwl/bartmann/Dokumente/nr61.pdf

Beer, J., Muschler, R., Kass, D. \& E. Somarriba (1998): Shade management in coffee and cacao plantations. - In: Agroforestry Systems 38: 139-164.

BundesverbäNDE NATURKost NATURWAREN (BNN) (2001):Trendbericht Naturkost2001.-http://62.112.68.138/ input/pdf/Trendbericht 2001.pdf

Boyce, J. et al. (1994): Sustenibilidad de la producción cafetalera costarricense y conveniencia del café orgánico como alternativa. - Facultatad para la economia y el medio ambiente de la Universidad Nacional de Heredia.

COMMISSION FOR ENVIRoNmental COOPERATION (CEC) (2000): Environmental and other labeling for coffee: The role of mutual recognition. - Ottawa: Communications and Public Outreach Department of the CEC Secretariat.

Commission for Environmental CoOperation (CEC) (1999): Measuring consumer interest in Mexican shadegrown coffee: An assessment of the Canadian, Mexican and US-markets. - Montréal: Communications and Public Outreach Department of the CEC Secretariat.

Corporación Educativo para el Desarollo CostarRICENSE (CEDECO) (1999): Estado actual de la agricultura orgánica en Costa Rica - Abschlussbericht. - San José.

Courville, S. (2001): Not just trade: Steps toward incorporating social and ecological costs into international trade. Lessons Learned From «Better» Case Studies on Production-to-Consumption Systems. Unveröffentlichte Dissertation, Faculty of Philosophy, Australian National University, Sydney.

EspinOZA, P.L. (1985): Untersuchungen über die Bedeutung der Baumkomponente bei agroforstwirtschaftlichem Kaffeeanbau an Beispielen aus Costa Rica. - = Göttinger Beiträge zur Land- und Forstwirtschaft in den Tropen und Subtropen 10, Göttingen.

EUROPÄISCHE WIRTSCHAFTSGEMEINSCHAFT (EWG) (1992): Verordnung Nr. 2092/91 des Rates vom 24. Juni 1991 über den ökologischen Landbau und die entsprechende Kennzeichnung der landwirtschafltichen Erzeugnisse und Lebensmittel, Brüssel.

FischersworRing, B. (2001): Guía para la caficultura ecológica.- Popayán, Eschborn: Deutsche Gesellschaft für Technische Zusammenarbeit GmbH (GTZ).

Galloway, G. \& J. BeER (1997): Oportunidades para fomentar la silvicultura en cafetales en América Central. - Turrialba: Centro Agrónomico Tropical de Investigación y Enseñanza /Deutsche Gesellschaft für Technische Zusammenarbeit GmbH (GTZ).

GerefFi, G. \& M. Korzewienicz (Hrsg.) (1994): Commodity chains and global capitalism. - Westport: Prager.

GiovanNUCCI, D. (2001): Sustainable coffee survey of the 
North American Specialty Coffee Industry. - Conducted for: The Summit Foundation, The Nature Conservancy, North American Commission for Environmental Cooperation, Specialty Coffee Association of America. - Report of the World Bank, Washington D.C.

Gomez-Pomba, A. (1997): Biodiversity and agriculture: Friends or foes? - In: Rice, R., Harris, A.M. \& J. McLEAN (Hrsg.) (1997): Proceedings of the $1^{\text {st }}$ Sustainable Coffee Congress, September 1996, Washington, D.C.: 1-17.

Gommans, C. (1996): From crop to cup - Costs and Benefits of Reducing Polluting Effects of Coffee. Unveröffentlichte Diplomarbeit, Economic Faculty, Erasmus University, Rotterdam.

Hopkins, T. \& I. Wallerstein (1994): Commodity chains: constructs and research. - In: GerefFI, G. \& M. Korzeniewicz (Hrsg.) (1994): Commodity chains and global capitalism. - Westport: Prager: 17-20.

International Trade Organization (ITC) (1999): Organic Food and Beverages: World Supply and Major European Markets. - Genf: UNCTAD, WTO; ITC. Luibrand, A. (1997): Biologisch angebauter Kaffee eine ökonomisch lohnende Alternative? Eine Untersuchung am Beispiel Costa Rica. - Unveröffentlichte Diplomarbeit, Landwirtschaftliche Fakultät der Universität Hohenheim.

Lynbaek, A. (2000): Productivity and profitability of multistrata organic versus conventional coffee farms. Unveröffentlichte Diplomarbeit, Centro Agrónomico Tropical de Investigación y Enseñanza, Turrialba.

MAYER, C. (2002): Umweltstandards im Weltkaffeehandel - eine institutionenökonomische Analyse der globalen Warenkette von Kaffee. - Unveröffentlichte Dissertation am Fachbereich Geographie der Phillipps-Universität, Marburg.

Muschler, R. (1999): Shade Improves the Quality of Coffee Arabica L. in a Suboptimal Coffee-zone of Costa Rica. - Arbeitspapier CATIE, Turrialba.

Muschler, R. (1997): Shade or sun for ecologically sustainable coffee production: A summary of environmental key factors. - III. Semana Científica del Centro Agrónomico Tropical de Investigación y Enseñanza (CATIE): 109-112.

Organic Coffee Association (ORCA) (2000): Internes Papier. - Camp Meeker, California.

Ponte, S. (2001): The «Latte Revolution»? Winners and losers in the restructuring of the global coffee marketing chain. - Centre for development research : CDR Working Paper, 01.3, June 2001, Kopenhagen.

Porter, R. (2000): Political-economic restructuring and Mexico's small coffee farmers. - In: TARDANICO, R. \& M. Rosenberg (2000): Poverty or development. - New York, London: Routledge: 111-139.

Raikes, P., Jensen, M.F. \& S. Ponte (2000): Global commodity chain analysis and the french filière approach: Comparison and critique. - CDR Working Paper 00.3, Kopenhagen.
Rice, P.D. \& J. McLean (1999): Sustainable coffee at the crossroads. - A white paper prepared for the Consumer's Choice Council, Washington, D.C.: http:/ /www.consumerscouncil.org/coffee/coffeebook/ coffee.pdf; 9.4.2003.

RICE, R. \& J.R. WARD (1997): El café, la conservación ambiental y el comercio en el hemisferio occidental. - Washington, D.C.: Natural Resource Defense Council and the Smithsonian Migratory Bird Center. http://natzoo.si.edu/smbc/Research/Coffee/coffee.htm Rodriguez, D. (2001): The Latin American Organic Coffee Industry: U.S. Market Inroads. - = Faculty Series 01-16, Department of Agricultural and Applied Economies, University of Georgia.

Rosen, S. et al. (2000): The U.S. Organic Market: Size, trends and implications of Central American agricultural exports. - Development Discussion Paper No. 737, Harvard University, Institute for International Development, Cambridge.

Stamm, A. (1999): Kaffeewirtschaft in Zentralamerika. Aktuelle Situation und Entwicklungsperspektiven. In: Geographische Rundschau 7-8: 399-407.

TALbot, J.M. (1997a): Where does your coffee dollar go? The division of income and surplus along the coffee commodity chain. - In: Studies of Comparative International Development, 32, 1: 56-91.

TALвот, J.M.(1997b): The struggle for control of a commodity chain. Instant coffee from Latin America. - In: Latin American Research Review 32: 117-135.

United Nations Conference on Trade and DevelopMENT (UNCTAD) (1993): Los efectos de la producción y transformación de productos básicos sobre el medio ambiente: síntesis de estudios monográficos sobre el arroz, el cacao y el café. - UNCTAD-Documento 2511, Genf.

United States Department of Agriculture (USDA) - Global Agriculture Information Network (2000): Gain Report of Costa Rica - Costa Rica coffee annual. - Prepared by P. Bertsch, US Embassy, San José, Costa Rica:htpp://www.fas.usda.gov/GainFiles/200208; Stand 10/2001.

United States Department of Agruculture (USDA) (2002):The National Organic Program;www.ams.usda.gov/ nop/NOP/NOPhome.html; Stand 01/2002; Stand 04/2003. VÁsQuez, A. (1999): Impacto ambiental del monocultivo café en Cotobrus. - Tesis de la Facultat para el medio ambiente, Universidad Estatal a Distancia, San Vito.

\section{Zusammenfassung: Können Öko-Siegel einen neuen Handlungsspielraum für kleinbäuerliche Kaffeepro- duzenten schaffen? - Das Beispiel Costa Rica}

Im letzten Jahrzehnt hat das Bestreben, soziale und ökologische Aspekte stärker in den Weltkaffeehandel $\mathrm{zu}$ integrieren, bedeutend zugenommen. Zum Einen, um die weltweit stetig wachsende Armut der Kaffee- 
bauern und zum Anderen, um die ökologische Belastung beim Kaffeeanbau zu mindern. Es entstanden Öko-Siegel, die über die Vermittlung neuer Nachfrageimpulse nach ökologisch erzeugtem Kaffee einen Lösungsbeitrag für die Dilemmasituation des Verarmungsprozesses und des steigenden Ressourcenverbrauchs im Kaffeesektor leisten sollen. Die Öko-Siegel wirken jedoch nicht isoliert, sondern sind eingebettet in ein institutionelles Geflecht von ökologischen, ökonomischen und politisch-institutionellen Rahmenbedingungen. Die hieraus entstehenden Wechselwirkungen bestimmen die Möglichkeiten und Grenzen der Kaffeebauern, den durch die Siegel geschaffenen Handlungsspielraum zu nutzen. Darüber hinaus hängt dies in hohem Maße von der Befähigung der Akteure $\mathrm{ab}$, die neu entstandenen Potenziale zu nutzen. Es zeigt sich, dass Umweltschutz und Wettbewerbsfähigkeit diejenigen Unternehmen am besten verbinden, die effektive Formen der Zusammenarbeit mit den vorund nachgelagerten Warenkettensegmenten sowie mit Akteuren und Organisationen außerhalb der Warenkette aufweisen.

\section{Summary: Can Organic Labels Create a New Field of Action for Small-Holder Coffee Producers? The example of Costa Rica}

During the last decade, the efforts to integrate social and ecological aspects into the global coffee trade have significantly increased. On the one hand, the aim is to alleviate the increasing poverty of the coffee farmers, on the other, to contribute towards reducing the negative impact of coffee cultivation on the ecology. Organic labels were created to generate new consumer needs for organically produced coffee as a means of contributing towards solving the problems of both increasing poverty and increasing resource demands in the coffee sector. The organic labels are not to be seen as isolated entities but rather as part of an institutional network governd by ecological, economic and political structures. The resulting interaction determines to what degree the coffee farmers are able to make use of the chances created by the organic label. The abilities and qualification of the actors also strongly influence the degree to which use is made of the potentials offered. Those companies that try to cooperate with segments before and after themselves in the product chain, as well as with actors and organisations outside the chain are best able to combine environmental protection with market competitiveness to their advantage.

\section{Résumé: Les labels écologiques peuvent-ils susciter un nouvel espace de manouvre pour les petits pro- ducteurs de café? L'exemple de Costa Rica}

Durant la décennie écoulée, la tendance vers une intégration plus importante de considérations sociales et écologiques au commerce mondial du café s'est affermie sensiblement. D'abord pour réduire la pauvreté croissante, à l'échelle mondiale, des paysans producteurs de café, puis pour diminuer les nuisances écologiqes lors de la mise en œuvre des plantations. Sont apparus des labels écologiques qui contribuèrent à renforcer la demande de café produit selon des normes écologiques, processus qui a permis de contrer la paupérisation et la destruction croissante de la ressource dans le secteur du café. Les labels écologiques n'agissent cependant pas de façon isolée; ils s'inscrivent dans un contexte institutionnel fondé sur un ensemble d'articulations écologiques, économiqes et politiques. Les relations réciproques qui en résultent déterminant lespossibilités et limites du paysannat producteur de café dans le cadre du nouvel espace de manœuvre consenti. Elles dépendent en grande partie de la qualification des acteurs et de leur aptitude à mettre à profit les potentialités offertes. Il apparaît que la protection de l'environnement et l'aptitude à la compétitivité favorisent surtout les entreprises à même de collaborer effectivement aux diverse formes de segments des chaînes de marchandises, ainsi qu'avec les acteurs et organisations extérieurs à la chaîne de marchandises.

\section{Didaktische Hinweise}

- Welches sind die innovativen Akteure entlang der Warenkette, die eine Anwendung von Öko-Siegeln forcieren?

- Welches sind Erfolgs- und Hemmfaktoren bzw. worin liegen die Potenziale und Grenzen bei der Anwendung von Öko-Siegeln im Kaffeesektor?

Inwieweit lassen sich diese Erfahrungen auf andere Agrarprodukte übertragen?

- Welchen Beitrag leisten die Öko-Siegel zu einer nachhaltigen Exportwirtschaft im Kaffeesektor?

- Mit welchen alternativen Strategien kann die Dilemma-Situation der Verarmung und des Ressourcenverbrauchs im Weltkaffeemarkt entschärft werden?

Claudia Mayer, Deutsche Gesellschaft für Technische Zusammenarbeit $\mathrm{GmbH}$, Programmbüro für Sozialund Ökostandards, Dag-Hammarskjöld-Weg 1-5, D-65760 Eschborn.

e-mail: claudia.mayer@gtz.de

\section{Manuskripteingang/received/manuscrit entré le} 20.11.2002

Annahme zum Druck/accepted for publication/accepté pour l'impression: 3.4 .2003 